\title{
Pericardial Effusion, CTCAE
}

National Cancer Institute

\section{Source}

National Cancer Institute. Pericardial Effusion, CT CAE. NCI Thesaurus. Code C143743.

A disorder characterized by fluid collection within the pericardial sac, usually due to inflammation. 